Voix et Images

voixetimages

\title{
Bibliographie de Guy Dufresne
}

\section{Aurélien Boivin et Lucie Robert}

Volume 9, numéro 1, automne 1983

Guy Dufresne

URI : https://id.erudit.org/iderudit/200421ar

DOI : https://doi.org/10.7202/200421ar

Aller au sommaire du numéro

\section{Éditeur(s)}

Université du Québec à Montréal

\section{ISSN}

0318-9201 (imprimé)

1705-933X (numérique)

Découvrir la revue

\section{Citer ce document}

Boivin, A. \& Robert, L. (1983). Bibliographie de Guy Dufresne. Voix et Images, 9(1), 59-81. https://doi.org/10.7202/200421ar d'utilisation que vous pouvez consulter en ligne.

https://apropos.erudit.org/fr/usagers/politique-dutilisation/ 


\title{
Bibliographie de Guy Dufresne
}

\author{
par Aurélien Boivin et Lucie Robert, Université Laval
}

\section{I - OEUVRES}

\section{A - $\dot{A}$ la radio}

Sacrifice. Radiothéâtre présenté au concours de Radio-Canada en 1945; diffusé à l'émission les Voix du pays, le 17 février 1946, dans une réalisation de Berthe Lavoie. (Sous le pseudonyme de Jacques DOLLIER.)

Le Contrebandier. Radiothéâtre présenté au concours de Radio-Canada en 1945; diffusé à l'émission les Voix du pays, le 10 mars 1946, dans une réalisation de Berthe Lavoie. (Sous le pseudonyme de Louis VARECH.)

En côtoyant la mer. Scènes d'un radiothéâtre diffusé par Radio-Canada, à l'émission les Voix du pays, le 4 août 1946, dans une réalisation de Judith Jasmin.

L'Histoire des vergers de Missisquoi. Dramatisation historique diffusée par Radio-Canada, à l'émission le Réveil rural, le 2 octobre 1946.

Le Ciel par-dessus les toits. Dramatisations historiques diffusées par RadioCanada, du 12 octobre 1947 au 24 avril 1955; dans une réalisation de Guy Mauffette :

- Monseigneur François de Laval : du 12 octobre au 9 novembre 1947; du 17 octobre au 15 novembre 1948; du 6 au 27 novembre 1949; du 4 novembre 1951 au 30 mars 1952.

- Marie de l'Incarnation : du 16 novembre au 7 décembre 1947; du 2 novembre 1952 au 5 avril 1953.

- Marguerite Bourgeoys : du 14 décembre 1947 au 4 janvier 1948; du 5 novembre 1950 au 25 mars 1951.

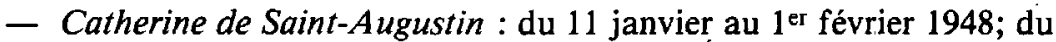
7 novembre 1954 au 27 mars 1955.

- Jeanne Mance : du 8 février au 28 mars 1948; du 5 au 26 février 1950; du 8 novembre 1953 au 16 mai 1954. 
- Histoire de Mai : du 21 novembre au 12 décembre 1948.

- L'île aux fraudes : du 26 décembre 1948 au 23 janvier 1949.

- Récit d'Angèle Nau : du 30 janvier au 27 février 1949.

- Jean de Brébeuf : du 6 au 13 mars 1949.

- La Souche à Mathurin : du 20 mars au 17 avril 1949.

- Le Bêcheur qui chantonnait : du 4 au 25 décembre 1949.

- Hoël dit le Barbu : du 15 au 29 janvier 1950.

- Loretta la Huronne : du 5 au 26 mars 1950.

- Mance-Acadie : 3 avril 1955.

- Laval-Acadie : du 10 au 17 avril 1955.

- Bourgeoys-Acadie : 24 avril 1955.

Deux-zéro-sonnez-quatre. Monologues diffusés par Radio-Canada, du 9 juillet au $1^{\text {er }}$ octobre 1951, dans une réalisation de Guy Beaulne.

Perrette et le trio des petits. Présentations de chansons et de fantaisies diffusées par Radio-Canada, du 4 octobre 1951 au 2 juillet 1953, dans une réalisation de Guy Mauffette.

Pêcheurs de Gaspésie. Radiothéâtre adapté d'un roman de Marie LeFranc, diffusé par Radio-Canada, à l'émission les Grands Romans canadiens, le 14 octobre 1951, dans une réalisation de Guy Beaulne.

Burins d'histoire. Dramatisations historiques diffusées par Radio-Canada, sur ondes courtes, en 1952, dans une réalisation de Judith Jasmin. (En collaboration avec Judith Jasmin.)

Le Choc des idées. Sketches préparés pour une émission d'information agricole, diffusés par Radio-Canada, du 7 janvier 1952 au 28 mars 1955, dans une réalisation de Marc Thibault.

Vie de Selkirk. Fondateur de la lère colonie manitobaine. Dramatisation historique diffusée par Radio-Canada, du 4 mars au $1^{\mathrm{er}}$ avril 1952, dans une réalisation de Marc Thibault.

Jean-François Pépinou. Chronique de Frelibourg. Chronique agricole diffusée par le Service international de Radio-Canada, d'avril à juin 1952, dans une réalisation de Judith Jasmin.

Le Trio des petits. Présentations de chansons et de fantaisies diffusées par le Service international de Radio-Canada, du 9 juin au 31 août 1952, dans une réalisation de Judith Jasmin.

Pierre de La Vérendrye. Dramatisation historique diffusée par RadioCanada, le 26 octobre 1952, à l'occasion de l'inauguration du réseau français de l'Ouest, dans une réalisation de Guy Mauffette. 
Allons tous vers l'étable. Fantaisie diffusée par Radio-Canada, le 24 décembre 1952, dans une réalisation de Guy Mauffette et de Lucien Côté.

À travers le pays. Émission spéciale diffusée par Radio-Canada, le 2 juin 1953, à l'occasion du Couronnement d'Élizabeth II, dans une réalisation de Lucien Côté.

Sondages. Émission spéciale diffusée par Radio-Canada, le 1er juillet 1953, à l'occasion des fêtes de la Confédération, dans une réalisation de Guy Mauffette.

Félix Poutré. Adaptation radiophonique du drame de Louis Fréchette, diffusée par Radio-Canada, à l'émission le Théâtre canadien, le 2 août 1953, dans une réalisation de Guy Beaulne.

Le Jeu de la voyagère. Adaptation radiophonique d'un texte dramatique de Rina Lasnier, diffusée par Radio-Canada, à l'émission le Théâtre canadien, le 16 août 1953, dans une réalisation de Guy Beaulne.

Perrette et Jean Merlot. Présentations de chansons et de fantaisies diffusées par Radio-Canada, du 1er octobre 1953 au 24 juin 1954, dans une réalisation de Roger de Vaudreuil.

Les Feux de la Saint-Jean. Dramatisation historique diffusée par RadioCanada, le 24 juin 1954, dans une réalisation de Guy Mauffette.

Perrette et ses Fredons. Présentations de chansons et fantaisies diffusées par Radio-Canada, du 7 octobre 1954 au 28 juin 1956, dans une réalisation de Roger de Vaudreuil.

Le Pinson. Dramatisation religieuse diffusée par Radio-Canada, le 8 décembre 1954, dans une réalisation de Guy Mauffette.

Légende de Noël. Radiothéâtre diffusé par Radio-Canada, le 25 décembre 1958.

Le Bêcheur qui chantonnait. Radiothéâtre diffusé par Radio-Canada, à l'émission Images du pays, le 16 octobre 1960, dans une réalisation de JeanRené Major.

Dieu ayde au premier baron chrétien. Radiothéâtre diffusé par RadioCanada, à l'émission Images du pays, le 23 octobre 1960, dans une réalisation de Jean-René Major.

Mon village sur la mer. Radioroman tiré de Cap-aux-Sorciers diffusé par Radio-Canada, du 26 février au 2 avril 1961, dans une réalisation de JeanRené Major.

Wahta. Radiothéâtre tiré de Ka-na-wio, diffusé par Radio-Canada, à l'émission Sur toutes les scènes du monde, le 23 mars 1964, dans une réalisation de Roger Citerne. 
Un train passe. Dramatisation religieuse diffusée par CKAC, en février et mars 1969, dans une réalisation de Jeannette Brouillet, d'après une conception de Jean-Guy Dubuc et de Guy Mauffette.

Le Cri de l'engoulevent. Radiothéâtre adapté du drame, diffusé par RadioCanada, à l'émission Sur toutes les scènes du monde, le 19 mars 1974, dans une réalisation d'Ollivier Mercier-Gouin.

Ce maudit Lardier. Radiothéâtre tiré des Forges de Saint-Maurice, diffusé par Radio-Canada, en juin 1976.

\section{B - À la télévision}

L'Île-aux-pommes ou la Hongroise. Téléthéâtre diffusé par Radio-Canada, à l'émission Théâtre, le 26 octobre 1952, dans une réalisation de Georges Groulx.

Nicolas Dumetz. Téléthéâtre diffusé par Radio-Canada, à l'émission Théâtre d'été, le $1^{\text {er }}$ septembre 1954, dans une réalisation de Jean-Paul Fugère.

(Les Sacrements.) Dramatisations religieuses diffusées par Radio-Canada, à l'émission Eaux vives, du 24 octobre 1954 au 20 février 1955, dans une réalisation de Claude Désorcy.

La Veilleuse. Téléthéâtre diffusé par Radio-Canada, à l'émission 30 secondes, le 24 décembre 1954, dans une réalisation de Fernand Doré.

Cap-aux-Sorciers. Téléroman diffusé par Radio-Canada, du 7 juin 1955 au 12 juin 1958, dans une réalisation de Maurice Leroux et de Paul Blouin.

Marie Hurdouil s'est noyée. Téléthéâtre diffusé par Radio-Canada, à l'émission Été 55, le 17 août 1955, dans une réalisation de Jean-Paul Fugère.

Propriétaire d'un jour. Téléthéâtre diffusé par Radio-Canada, à l'émission les Jeunes Auteurs, le 21 juillet 1958.

À la romance. Présentations de chansons et de textes de variétés diffusés par Radio-Canada, du 11 octobre 1958 au 30 mai 1959, dans une réalisation de Roger Barbeau et Lisette LeRoyer.

Kébec. Téléthéâtre diffusé par Radio-Canada, le 2 novembre 1958, à l'occasion du $350^{\mathrm{e}}$ anniversaire de la fondation de Québec, dans une réalisation de Jean-Paul Fugère.

Mesure de guerre. Téléthéâtre diffusé par Radio-Canada, à l'émission Première, le $1^{\text {er }}$ mars 1960, dans une réalisation de Paul Blouin.

Chemin privé. Téléthéâtre adapté du Cri de l'engoulevent, diffusé par Radio-Canada, le 17 novembre 1960, dans une réalisation de Jean-Paul Fugère. 
Les Traitants. Téléthéâtre diffusé par Radio-Canada, à l'émission Thêâtre du dimanche, le 15 janvier 1961, dans une réalisation de Jean Dumas.

Les Pléiades. Dramatique pour enfants diffusée par Radio-Canada, à l'émission le Grand Duc, le 26 janvier 1961, dans une réalisation de Pierre Leboeuf, Pierre Monette et Charles Dumas.

Kah-na-wio ou la Rivière aux belles chutes. Téléroman diffusé par RadioCanada, du 15 octobre 1961 au 8 juillet 1962, dans une réalisation d'Aimé Forget.

Comme tu me veux. Téléthéâtre adapté d'un texte du Luigi Pirandello, diffusé par Radio-Canada, le 7 octobre 1962, dans une réalisation de JeanPaul Fugère.

Les Trois sœeurs. Téléthéâtre adapté d'un texte d'Anton Tchékhov, diffusé par Radio-Canada, le 12 mai 1963, dans une réalisation de Paul Blouin.

Septième nord. Téléroman diffusé par Radio-Canada, du 29 septembre 1963 au 30 août 1967, dans une réalisation d'André Bousquet.

Des souris et des hommes. Téléthéâtre adapté d'un roman de John Steinbeck, diffusé par Radio-Canada, le 17 janvier 1971, dans une réalisation de Paul Blouin.

Les Forges de Saint-Maurice. Téléroman diffusé par Radio-Canada, du 4 septembre 1972 au 19 mai 1975, dans une réalisation de Louis Bédard et Richard Martin.

Johanne et ses vieux. Téléthéâtre diffusé par Radio-Canada, à l'émission les Beaux Dimanches, le 24 octobre 1976, dans une réalisation de Richard Martin.

Ces dames de l'estuaire. Téléthéâtre adapté d'un texte de Edward Percy et Reginald Denham, diffusé par Radio-Canada, -à l'émission les Beaux Dimanches, le 9 avril 1978, dans une réalisation de Paul Blouin.

Décembre. Téléthéâtre diffusé par Radio-Canada, à l'émission les Beaux Dimanches, le 17 décembre 1978, dans une réalisation de Jean-Paul Fugère.

Aéroport: court-circuit. Téléthéâtre diffusé par Radio-Canada, à l'émission les Beaux Dimanches, le 27 mars 1983, dans une réalisation de Jean-Claude Labrecque.

\section{C - Au cinéma}

Marguerite Bourgeoys. Texte préparé pour l'Office du film du Québec à l'occasion de la béatification de Marguerite Bourgeoys, 1954.

Louis-Joseph Papineau, le demi-dieu. Scénario préparé pour l'Office national du film du Canada à l'occasion du centenaire de la Confédération canadienne, produit par Julien Bigras, 1965. $26 \mathrm{~min}$. 
Les Ordres. Dialogues du film de Michel Brault, produit par les Productions Prisma Inc., 1974. $107 \mathrm{~min}$.

\section{D - À la scène}

Le Jeu sur la presqu't̂le. Jeu dramatique créé au Parc Lafontaine à Montréal, le 22 juin 1952, dans une mise en scène de Fernand Doré.

Le Cri de l'engoulevent. Drame créé à la Comédie canadienne, le 26 janvier 1960, dans une mise en scène de Jan Doat; reprise par le Théâtre populaire du Québec, dans une mise en scène d'Albert Millaire, en tournée québécoise en mars et avril 1970. Une deuxième version de la pièce fut mise en scène par Albert Millaire pour la Nouvelle Compagnie théâtrale et créée le 16 octobre 1971.

Docile. Comédie créée à la Comédie canadienne, le 10 mai 1968, dans une mise en scène de Gratien Gélinas.

Les Traitants. Drame historique créé par le Théâtre du Nouveau Monde à la salle Port-Royal de la Place des Arts de Montréal, le 2 mars 1969, dans une mise en scène d'Albert Millaire.

La Moscheta. Traduction de la pièce de Angelo Beolco dit Ruzante, créée par la Nouvelle Compagnie théâtrale, le 8 janvier 1974, dans une mise en scène de Claude Deslandes.

Jean Besset. Drame historique créé au Parc historique national Fort Chambly, le 30 mai 1974.

Un tramway nommé Désir. Traduction et adaptation de la pièce de Tennessee Williams, créée par la Cie Jean Duceppe, à la salle Port-Royal de la Place des Arts de Montréal, le $1^{\text {er }}$ octobre 1974, dans une mise en scène d'Olivier Reichenbach.

\section{E - Publications}

Le Ciel par-dessus les toits : «En hommage à François de Montmagny (sic) Laval, premier évêque de Québec», l'Action nationale, vol. XXXIX, $\mathrm{n}^{\circ} 3$ (avril 1952), pp. 189-213; "(En hommage à Jeanne Mance)», dans RolandJ. Auger, la Grande Recrue de 1653, préface de Joseph-Papin Archambault, Montréal, Société généalogique canadienne-française, 1955, 205 p. (v. pp. 161-193).

«Dialogue sur le théâtre», la Barre du jour, vol. I, nos 3-4-5 (juilletdécembre 1965), pp. 62-64.

Cap-aux-Sorciers. I. Fabienne, Montréal, Leméac, 1969, 268 p. Photos. (Les Beaux Textes).

Le Cri de l'engoulevent, notes préliminaires d'Alain Pontaut, Montréal, Leméac, 1969, 123 p. Ill. (Théâtre canadien, no 9); The Call of the 
Whippoorwill. Translated by Philip London et Laurence Bérard, introduction by Alain Pontaut, Toronto, New Press, 1972, xiv, 102 p. (New Drama, $\mathrm{n}^{\circ}$ 2); un extrait parut dans Jan DOAT, Anthologie du théâtre québécois, 1606-1970, Québec, les Éditions La Liberté, 1973, 505 p. (v. pp. 323-326).

Les Traitants, Montréal, Leméac, 1969,176 p. (Théâtre canadien, $\mathrm{n}^{\circ} 8$ ); un extrait parut dans Archives des lettres canadiennes, tome $\mathrm{V}$, le Théâtre canadien-français, Montréal, Fides, 1976, 1005 p. (v. pp. 777-778).

«Un texte, deux traductions (Des souris et des hommes - extrait)», les Cahiers de la Nouvelle Compagnie théâtrale, vol. V, $\mathrm{n}^{\circ} 1$ (octobre 1970), pp. 20-22.

"Guy Dufresne par lui-même», les Cahiers de la Nouvelle Compagnie théâtrale, vol. VI, $\mathrm{n}^{\circ} 1$ (octobre 1971), pp. 4-6.

Docile. Comédie en deux actes, Montréal, Leméac, 1972, 103 p. (Répertoire québécois, nos 18-19).

«Guy Dufresne présente sa Moscheta (suivi d'un extrait)», les Cahiers de la Nouvelle Compagnie théâtrale, vol. VIII, ${ }^{\circ} 2$ (janvier 1974), pp. 22-23.

Ce maudit Lardier, préface de Maurice Filion, Montréal, Leméac, 1975, xiii (4) 167 p. (Théâtre, $n^{\circ} 49$ ).

\section{II - ÉTUDES}

\section{Généralités}

(Anonyme), "Au sujet de Guy Dufresne, l'auteur de Ka-na-wi-o (sic)», le Droit, vol. L, $\mathrm{n}^{\circ} 23$ (27 janvier 1962), p. 5.

, «Le ministère des Affaires culturelles distribue $\$ 155,000$ à 58 boursiers", la Presse, vol. LXXXIII, nº 69 (23 mars 1967), p. 26.

«L'enfance de Guy Dufresne», Ici Radio-Canada, vol. IV, $n^{\circ} 32$ (1 $1^{\text {er-8 }}$ août 1970), p. 23.

, «(Entrevue)», les Cahiers de la Nouvelle compagnie théâtrale, vol. VI, $\mathrm{n}^{\circ} 1$ (octobre 1971), pp. 8, 10, 12, 18, 23.

, "Guy Dufresne», Archives des lettres canadiennes, tome V, le Théâtre canadien-français, Montréal, Fides, 1976, 1005 p. (v. pp. 776-778). juin -4 juillet 1980 ), p. 8 . "Chez Guy Dufresne», Ici Radio-Canada, vol. XIV, nº 27 (28

CANTIN, Pierre, Normand HARRINGTON et Jean-Paul HUDON, Bibliographie de la critique de la littérature québécoise dans les revues des $X I X^{\mathrm{e}}$ et $X X^{\mathrm{e}}$ siècles, Ottawa, Centre de recherche en civilisation canadienne-française, 1979, 5 vols. : x, 1254 p. (pagination continue). (Documents de travail du CRCCF, $\mathrm{n}^{\circ} 12$ ) (v. vol. III, pp. 430-433). 
G(ERMAIN), J(ean)-C(laude), «Le secret de Guy Dufresne : 'Je me suis penché sur la vie'», le Petit Journal, vol. XLII, n 25 (14 avril 1968), p. 57.

GRANDPRÉ, Pierre de (directeur), Histoire de la littérature française du Québec, tome IV, Roman, théâtre, histoire, journalisme, essai, critique (de 1945 à nos jours), Montréal, Librairie Beauchemin limitée, 1969, 428 p. (v. pp. 229-230).

GRUSLIN, Adrien, "Théâtre. Place aux adaptateurs», le Devoir, vol. LXV, n ${ }^{\circ} 96$ (27 avril 1974), p. 13.

HAMEL, Réginald, John HARE et Paul WYCZYNSKI, Dictionnaire pratique des auteurs québécois, Montréal, Fides, 1976, 723 p. (v. pp. 221-222).

LEGRIS, Renée, avec la collaboration de Suzanne ALLAIRE-POIRIER, Louise BLOUIN et Pierre PAGÉ, Dictionnaire des auteurs du radiofeuilleton québécois.

NÖ̈L, Claire, "Guy Dufresne. Poète de la mer... et maire de Frelighsburg», le Petit Journal, vol. XXXIV, nº 2 (8 novembre 1959), p. 109.

PAGÉ, Pierre et Renée LEGRIS, Répertoire des dramatiques québécoises à la télévision, 1952-1977, Montréal, Fides, 1977, 252 p. (Archives québécoises de la radio et de la télévision) (v. pp. 76-77, 140-142).

PAGÉ, Pierre, avec la collaboration de Renée LEGRIS et Louise BLOUIN, Répertoire des oeuvres de la littérature radiophonique québécoise, 1930-1970, Montréal, Fides, 1975, 826 p. (Archives québécoises de la radio et de la télévision) (v. pp. 266-276).

PONTAUT, Alain, Dictionnaire critique du théâtre québécois, Montréal, Leméac, 1972, 161 p. (v. pp. 47-49).

RINFRET, Édouard-G., le Théâtre canadien d'expression française. Répertoire analytique des origines à nos jours, préface de Robert Choquette, tome I, Montréal, Leméac, 1975, xxiii, 390 p. (v. pp. 356-364); tome IV, Montréal, Leméac, 1978, 338 p. (v. pp. 89-94).

UNION DES ÉCRIVAINS QUÉBÉCOIS, Dictionnaire des écrivains québécois contemporains, recherche et rédaction par Yves Légaré, Montréal, Québec/Amérique, 1983, 399 p. (v. pp. 139-140).

\section{Aéroport : court-circuit}

(Anonyme), «Un suspense remarquable», Ici Radio-Canada, vol. XVII, ${ }^{\circ}$ 13 (26 mars-1 ${ }^{\text {er }}$ avril 1983), p. 4.

, «Spéciaux et premières», T.V. Hebdo, vol. XXIV, n 13 (26 mars-1 ${ }^{\text {er }}$ avril 1983), p. 5-A. 
BASILE, Jean, «Aéroport : court-circuit», le Devoir, vol. LXXIV, $n^{\circ} 73$ (29 mars 1983), p. 9.

BERNIER, Yves, «Court-circuit ou Comment manquer son coup», le Soleil, vol. LXXXVII, $\mathrm{n}^{\circ} 77$ (28 mars 1983), p. A-10.

BISSONNETTE, Lise, «De la culture des navets», le Devoir, vol. LXXIV, $\mathrm{n}^{\circ} 76$ (2 avril 1983), p. 12.

COUSINEAU, Louise, "Court-circuit : grande noirceur dramatique à Radio-Canada», la Presse, vol. XCIX, nº 74 (29 mars 1983), p. B-4.

LAROCHETTE, Réal, «La politique des navets», le Devoir, vol. LXXIV, $n^{\circ} 82$ (11 avril 1983), p. 10.

\section{Le Bêcheur qui chantonnait}

(Anonyme), "Images du Canada. Deux textes de Guy Dufresne», la Semaine à Radio-Canada, vol. XI, $\mathrm{n}^{\circ} 3$ (15-21 octobre 1960), p. 2.

Cap-aux-Sorciers (Voir aussi Mon village sur la mer)

(Anonyme), "Cap-aux-Sorciers le mardi soir, à Radio-Canada», le Droit, vol. XLIII, ${ }^{\circ} 134$ (11 juin 1955), p. 8.

, «Contes, légendes et chansons dramatisés par Guy Dufresne», la Semaine à Radio-Canada, vol. $\mathrm{V}, \mathrm{n}^{\circ} 35$ (5-11 juin 1955), p. 8.

, «Cap-aux-Sorciers», la Semaine à Radio-Canada, vol. VIII, $\mathrm{n}^{0} 2$ (12-18 octobre 1957); p. 11.

, «Cap-aux-Sorciers, le titre de noblesse de Guy Dufresne», la Semaine à Radio-Canada, vol. VIII, $\mathrm{n}^{\circ} 9$ (30 novembre - 6 décembre 1957), p. 8.

, «Cap-aux-Sorciers», le Livre canadien, vol. $\mathrm{I}, \mathrm{n}^{\circ} 72$ (1970).

, «Livres canadiens récents. Cap-aux-Sorciers», l'Église canadienne, vol. III, $\mathrm{n}^{\circ} 6$ (juin 1970), p. 199.

, «Cap-aux-Sorciers. Une des plus populaires œuvres de Guy Dufresne», Ici Radio-Canada, vol. VIII, no 13 (23-29 mars 1974), p. 3.

ALLARD, André, Luc DESCHÊNE et Claude LAPIERRE, «De Cap-auxSorciers aux Pays d'en-haut : les continuités (sic)», Chantiers, vol. XV, ${ }^{\text {os }}$ 5-6 (février-mars 1959), pp. 7-8 (v. p. 8).

BENOÎT, Jean, «Radio et télévision. Une explosion de colère à Cap-auxSorciers", le Devoir, vol. XLVIII, nº 61 (14 mars 1957), p. 9.

COUCKE, Paul, «Le fleuve, personnage principal d'un téléroman : Capaux-Sorciers», la Patrie, vol. XXII, n 45 (5 novembre 1956), p. 91.

DESBIENS, Lucien, "Ces échanges .'culturels' avec Moscou», Notre Temps, vol. XIII, $\mathrm{n}^{\circ} 34$ (7 juin 1958), p. 4. 
DUHAMEL, Roger, «Sur notre écran de télévision», l'École canadienne, vol. XXXII, $\mathrm{n}^{\circ} 3$ (novembre 1956), pp. 148-150 (voir p. 150).

GYL (pseudonyme), «Deux nouvelles émissions : Cap au sorcier (sic), la Rigolade», le Devoir, vol. XLVI, n 138 (18 juin 1955), p. 13.

HÉNAULT, Gilles, “'J'ai découvert mes personnages dans la réalité' Guy Dufresne», le Devoir, vol. LII, n 51 (2 mars 1961), p. 8.

LEGRIS, Renée, «Cap-aux-Sorciers», Dictionnaire des aeuvres littéraires du Québec, sous la direction de Maurice Lemire avec la collaboration de Gilles Dorion, André Gaulin, Alonzo Le Blanc, Aurélien Boivin, Roger Chamberland, Kenneth Landry et Lucie Robert, tome III, 1940-1959, Montréal, Fides, 1982, p. 154-157.

RENÉ DE COTRET, Marc, «Télé-critique (...) Cap-aux-Sorciers», la Patrie, vol. LXXIX, $\mathrm{n}^{\circ} 54$ (1 ${ }^{\text {er }}$ mai 1957), p. 6.

, «Télé-critique (...) Cap-aux-Sorciers», la Patrie, vol. LXXIX, $\mathrm{n}^{\circ} 66$ (15 mai 1957), p. 6.

, "Télé-critique. Cap-aux-Sorciers», la Patrie, vol. LXXIX, $\mathrm{n}^{\circ}$ 100 (26 juin 1957), p. 6.

, «Télé-critique. Cap-aux-Sorciers», la Patrie, vol. LXXIX, $\mathrm{n}^{\circ}$ 195 (18 octobre 1957), p. 6.

, «Télé-critique (...) Cap-aux-Sorciers», la Patrie, vol. LXXIX, $\mathrm{n}^{\circ} 207$ (1 $^{\mathrm{er}}$ novembre 1957), p. 6.

RIGAUD, Jean-Marc, «Présence de l'âme : Cap-aux-Sorciers», les Cahiers de la Nouvelle Compagnie théatrale, vol. VI, $\mathrm{n}^{\circ} 1$ (octobre 1971), p. 29.

VERGOR (pseudonyme), "Les rumeurs de la ville. Cap-aux-Sorciers», la Patrie, vol. LXXVII, $\mathrm{n}^{\circ} 78$ (31 mai 1955), p. 14.

Ce maudit Lardier (Voir aussi les Forges de Saint-Maurice)

(Anonyme), «Ce maudit Lardier», Ici Radio-Canada/radio, n ${ }^{\circ} 212$ (29 mai 1976), p. 6.

(septembre 1976).

«Ce maudit Lardier», le Livre canadien, vol. VII, $\mathrm{n}^{\circ} 253$

FILION, Maurice, Préface de la pièce, $p$. vii-xiii.

\section{Chemin privé (Voir aussi le Cri de l'engoulevent)}

(Anonyme), «Un téléthéâtre canadien. Chemin privé de Guy Dufresne», la Semaine à Radio-Canada, vol. XI, n 7 (12-18 novembre 1960), p. 2. , «Ils se rencontreront dans un Chemin privé», T.V. Hebdo, vol. I, $\mathrm{n}^{\circ} 14$ (17-23 novembre 1960), p. 25. 
HOFFMAN, Pierre, «Théâtre canadien d'expression française et réalisation télévisée». Thèse de maîtrise ès arts, Montréal, Université de Montréal, $1970, \mathrm{ii}, 108,6 \mathrm{f}$.

\section{Le Ciel par-dessus les toits}

(Anonyme), «Le Ciel par-dessus les toits», Radiomonde, vol. IX, n 46 (25 octobre 1947), p. 11.

mars 1948), p. 2.

, «Le Ciel par-dessus les toits», Radiomonde, vol. X, $\mathrm{n}^{\circ} 14(13$

, «Le Ciel par-dessus les toits revient sur les ondes», Radiomonde, vol. X, no 46 (23 octobre 1948), p. 3.

, «Le Ciel par-dessus les toits rend hommage à Mgr de Laval», la Semaine à Radio-Canada, vol. II, $n^{0} 4$ (4-10 novembre 1951), p. 4.

, «Haute appréciation de l'émission sur Monseigneur de Laval», la Semaine à Radio-Canada, vol. II, n 12 (30 décembre 1951 - 5 janvier 1952), p. 3 .

, «Comment une sainte vécut chez nous il y a 300 ans», le Droit, vol. XL, $\mathrm{n}^{\circ} 256$ (31 octobre 1952), p. 21.

, «La vie de Marie de l'Incarnation», la Semaine à RadioCanada, vol. III, $\mathrm{n}^{\circ} 7$ (23-29 novembre 1952), p. 3.

, «Huguette Oligny devant son rôle de Marie de l'Incarnation», Radiomonde, vol. XV, $\mathrm{n}^{\circ} 3$ (20 décembre 1952), p. 10.

, «L'histoire d'une jeune fille de la Nouvelle-France», la Semaine à Radio-Canada, vol. III, n 19 (15-21 février 1953), p. 3.

«10" saison du Ciel par-dessus les toits», la Semaine à RadioCanada, vol. IV, n $^{\circ} 4$ (1 $1^{\mathrm{er}}-7$ novembre 1953), p. 1; reproduit dans Radiomonde et Télémonde, vol. XV, $\mathrm{n}^{\circ} 48$ (31 octobre 1953), p. 24.

, «Figure attachante d'une héroïne des débuts de la colonie», la Semaine à Radio-Canada, vol. V, nº 6 (14-20 novembre 1954), p. 1.

, «Le Ciel par-dessus les toits évoquera les pages glorieuses de

l'histoire des Acadiens"), la Semaine à Radio-Canada, vol. V, n 26 (3-9 avril 1955), p. 3.

DUFRESNE, Guy, «L'histoire guide l'auteur du Ciel par-dessus les toits», la Semaine à Radio-Canada, vol. I, n 5 (12-18 novembre 1950), p. 2.

GAGNON, Denys, «Le Ciel par-dessus les toits», Dictionnaire des ceuvres littéraires du Québec, sous la direction de Maurice Lemire avec la collaboration de Gilles Dorion, André Gaulin, Alonzo Le Blanc, Aurélien Boivin, Roger Chamberland, Kenneth Landry et Lucie Robert, tome III, 1940-1959, Montréal, Fides, 1982, p. 196. 
LAURENDEAU, André, «Le Ciel par-dessus les toits», le Devoir, vol. XLIII, n ${ }^{\circ} 19$ (23 janvier 1952), p. 4.

, «Sur un texte de Guy Dufresne», l'Action nationale, vol. XXXIX, no 3 (avril 1952), p. 187-188.

\section{Le Contrebandier}

(Anonyme), «D'une heure à l'autre», le Droit, vol. XXXIV, n ${ }^{\circ} 58$ (9 mars 1946), p. 10; reproduit sous le titre «Le Contrebandier de Louis Varech au concours littéraire», Radiomonde, vol. VIII, $\mathrm{n}^{\circ} 13$ (9 mars 1946), p. 7.

, «Soirée unique à la radio. Six vainqueurs au concours littéraire de Radio-Canada», le Canada, vol. XLIV, ${ }^{\circ} 10$ (15 avril 1946), p. 11, 6; reproduit sous le titre «Proclamation des gagnants du concours de RadioCanada", le Devoir, vol. XXXVII, $\mathrm{n}^{\circ} 88$ (15 avril 1946), p. 4, et sous le titre «Clôture du concours de Radio-Canada», le Droit, vol. XXXIV, nº 90 (16 avril 1946), p. 4.

, «Le concours littéraire de Radio-Canada», Radiomonde, vol. VIII, $\mathrm{n}^{\circ} 19$ (20 avril 1946), p. 6.

\section{Le Cri de l'engoulevent (Voir aussi Chemin privé)}

(Anonyme), "Première à la Comédie canadienne. Le Cri de l'engoulevent, une pièce de G. Dufresne», la Presse, vol. LXXVI, n ${ }^{\circ} 70$ (6 janvier 1960), p. 40.

«Le théâtre. L'engoulevent, son cri... ce qu'en dit Guy Dufresne», la Presse, vol. LXXVI, nº 79 (16 janvier 1960), p. 30; reproduit dans le Devoir, vol. LI, no 13 (25 janvier 1960), p. 9.

1960), p. 2 et p. 4.

«Le théâtre», Bulletin du Cercle juif, vol. VI, n-51 (janvier

, «Création le 26. Le Cri de l'engoulevent se passe sur une ferme des Cantons de l'Est», la Presse, vol. LXXVI, n 73 (9 janvier 1960), p. 30. , «À la Comédie canadienne. Succès du nouveau système d'alternance», le Devoir; vol. LI, n 30 (13 février 1960), p. 12.

, «Succès ininterrompu du Cri de l'engoulevent», la Presse, vol. LXXVI, n ${ }^{\circ} 117$ (1 ${ }^{\mathrm{er}}$ mars 1960), p. 14.

, «Dernières représentations. Le Cri de l'engoulevent», le Devoir, vol. LI, $\mathrm{n}^{\circ} 55$ (14 mars 1960), p. 9.

, «Le monde et la ville. Le Cri de l'engoulevent», La Revue moderne, vol. XLI, n 12 (avril 1960), p. 10.

, «Une pièce de Guy Dufresne en tournée», la. Presse, vol. LXXXVI, n ${ }^{\circ} 54$ (5 mars 1970), p. 25. 
«Le 24 à la salle François-Brassard. Une pièce de Guy Dufresne. Le Cri de l'engoulevent", Progrès-dimanche, vol. LXXXII, n ${ }^{\circ} 2$ (8 mars 1970), p. 39; reproduit sous le titre «Le Cri de l'engoulevent joué par le TPQ», l'Action, vol. LXII, n 19028 (14 mars 1970), p. 15, et sous le titre «Le Cri de l'engoulevent clôture la saison théâtrale», le Nouvelliste, vol. $\mathrm{L}, \mathrm{n}^{\circ} 129$ (1 ${ }^{\mathrm{er}}$ avril 1970$)$, p. 16.

, «Troisième spectacle du TPQ à Sherbrooke. Peinture tragique de l'âme québécoise : Le Cri de l'engoulevent, de Guy Dufresne", la Tribune, vol. LXI, n² 26 (21 mars 1970), p. 19.

, «Le Théâtre populaire du Québec sera à Joliette», l'Action populaire, vol. LVIII, $\mathrm{n}^{\circ} 14$ (8 avril 1970), cahier 2, p. 1.

"Le Cri de l'engoulevent à l'Escale», le Droit, vol. LVIII, n' 24 (24 avril 1970), p. 19.

, «L Cri de l'engoulevent ouvrira la saison de la Nouvelle Compagnie théâtrale», l'Action, vol. LXIV, no 29 (9 octobre 1971), p. 12; reproduit sous le titre «À la NCT. Le Cri de l'engoulevent de Guy Dufresne», le Devoir, vol. LXII, n 233 (9 octobre 1971), p. 16.

«Le Cri de l'engoulevent de Guy Dufresne», Ici RadioCanada/radio, nº 97 (16-23 mars 1974), p. 22.

AROICHANE, Foutna, "Le père dans le théâtre québécois moderne». Thèse de maîtrise ès arts, Montréal, Université de Montréal, 1973, $256 \mathrm{f}$. (voir f. 103-113).

BARETTE, Andrée, «Place au théâtre», Progrès-dimanche, vol. LXXXII, n $^{\circ} 5$ (29 mars 1970), p. 44.

B(EAULIEU), M(ichel), «À la Nouvelle Compagnie théâtrale : le Cri de l'engoulevent de Guy Dufresne», Point de mire, vol. III, ${ }^{\circ} 4$ (6 novembre 1971), p. 39.

B(ÉRAUD), J(ean) (pseudonyme de Jacques LAROCHE), «Ả la Comédie canadienne. Pièces de MM. Gélinas, Toupin et Dufresne en système d'alternance», la Presse, vol. LXVI, nº 81 (19 janvier 1960), p. 35.

, «À la Comédie canadienne. Un nouvel auteur dramatique nous est né», la Presse, vol. LXXVI, nº 88 (27 janvier 1960), pp. 36-37.

BERTHIAUME, René, «Une pièce où l'on parle plus qu'on ne vit vraiment», la Tribune, vol. LXI, n³5 (2 avril 1970), p. 16.

BRISSENDEN, Connie, «Up the new!», Canadian Literature, $\mathrm{n}^{\circ} 59$ (Winter 1974), pp. 111-113.

CLOUTIER, Normand, «Selon Jan Doat : 'Guy Dufresne, c'est un peu Tchékhov...'», le Petit Journal, vol. XXXIV, nº 13 (24 janvier 1960), p. 91. 
DANDURAND, Renée-B., «1959 : le Cri de l'engoulevent. Demain la révolution tranquille», les Cahiers de la Nouvelle Compagnie théâtrale, vol. VI, $\mathrm{n}^{\circ} 1$ (octobre 1971), pp. 9-13.

DASSYLVA, Martial, «Sept-îles sait faire... même des lancements de livres. Le Cri de l'engoulevent», la Presse, vol. LXXXV, $\mathrm{n}^{\circ} 237$ (11 octobre 1969), p. 36.

, «Entre la pochade et le mélo», la Presse, vol. LXXXVII, $\mathrm{n}^{\circ} 242$ (19 octobre 1971), p. D-11.

D'AUTEUIL, Georges-Henri, «Le théâtre. Des jeux et hasards de l'amour», Relations, $\mathrm{n}^{\circ} 232$ (avril 1960), pp. 100-101. , «Théâtre d'ici», Relations, n 366 (décembre 1971), p. 342.

DESJARDINS, Maurice, «Debut of new Playwright. Le Cri de l'engoulevent», The Gazette, vol. CLXXXII (January 27, 1960), p. 14.

FÉRAL, Josette, «Le Cri de l'engoulevent de Guy Dufresne ou le conflit des grandes puissances», Archives des lettres canadiennes, tome V, le Théâtre canadien-français, Montréal, Fides, 1976, 1005 p. (v. pp. 667-673).

GARON, Jean «Le Cri de l'engoulevent, une tragédie bien mince», le Soleil, vol. LXXIII, $\mathrm{n}^{\circ} 65$ (16 mars 1970), p. 15.

GAY, Paul, «Le Cri de l'engoulevent», le Droit, vol. LVIII, $\mathrm{n}^{\circ} 25$ (25 avril 1970), p. 7.

GODARD, Barbara, «The Call of the Whippoorwill», Open Letter, second series, $n^{\circ} 7$ (Spring 1974), pp. 86-91.

GODIN, Jean-Cléo, «Les Traitants, Le Cri de l'engoulevent de Guy Dufresne», Livres et Auteurs Québécois, 1969, pp. 76-77.

HELLER, Zelda, «Revival of Dufresne Play Disappointing», The Montréal Star, vol. CIII, n 243 (October 18, 1971), p. 57.

HÉNAULT, Gilles, “À la Comédie canadienne. Le Cri de l'engoulevent», le Devoir, vol. LI, $\mathrm{n}^{\circ} 16$ (28 janvier 1960), p. 7.

KEMPF, Georges-Marcel, dit Yerri, «D'un salon parisien à une cour de ferme canadienne», Cité libre, vol. XI, n 25 (mars 1960), p. 30; reproduit dans les Trois coups à Montréal. Chroniques dramatiques 1959-1964, Montréal, Librairie Déom, 1965, 383 p. (voir pp. 326-327).

, «Chroniques du temps perdu. Théâtre et société à Montréal», Cité libre, vol. XV, $\mathrm{n}^{\circ} 78$ (juillet 1965), pp. 27-29.

LACROIX, Lucien, "À la Comédie canadienne. Le Cri de l'engoulevent (pièce en trois actes de Guy Dufresne)», le Quartier latin, vol. XLII, $\mathrm{n}^{\circ} 32$ (2 février 1960), p. 4. 
LASNIER, Marie, «L'univers théâtral de Guy Dufresne». Thèse de maîtrise ès arts, Montréal, McGill University, 1977, iii,167 f.

LE BLANC, Alonzo, «Y a-t-il une tragédie québécoise?», Nord, $\mathrm{n}^{\text {os }} 4-5$ (automne 1972 - hiver 1973), pp. 93-110 (voir p. 98).

, «Le Cri de l'engoulevent», Dictionnaire des oeuvres littéraires du Québec, sous la direction de Maurice Lemire avec la collaboration de Gilles Dorion, André Gaulin, Alonzo Le Blanc, Aurélien Boivin, Roger Chamberland, Kenneth Landry et Lucie Robert, tome IV, 1960-1969, Montréal, Fides, 1984 (à paraître).

LEROUX, Normand, «La littérature québécoise contemporaine. 1960-1977. III. Le théâtre», Études françaises, vol. XIII, nos 3-4 (octobre 1977), pp. 339-363 (voir p. 347).

MALTAIS, Murray, «Le Cri de l'engoulevent. Quand un texte n'est ni chair ni poisson», le Droit, vol. LVIII, n 26 (27 avril 1970), p. 11.

MARSOLAIS, Gilles, «Gilles Pelletier, metteur en scène du Cri de l'engoulevent», les Cahiers de la Nouvelle Compagnie théâtrale, vol. VI, $\mathrm{n}^{\circ} 1$ (octobre 1971), p. 26.

MELANÇON, Joseph, "Un drame décentré», les Cahiers de la Nouvelle Compagnie théâtrale, vol. VI, n ${ }^{\circ} 1$ (octobre 1971), pp. 19-21.

MORISSETTE, Julien, «Le Cri de l'engoulevent. Au moins un acte d'excellent théâtre», Notre Temps, vol. XV, $\mathrm{n}^{\circ} 17$ (6 février 1960), p. 4.

PONTAUT, Alain, Présentation de la pièce, pp. 7-19.

RIALLAND, Yvonne, «À voir et à entendre cette semaine dans la métropole», Notre Temps, vol. XIV, $n^{\circ} 50$ (26 septembre 1959), p. 6.

RICHER, Julia, «Guy Dufresne aime les thèmes forts», Notre Temps, vol. XVI, n 7 (26 novembre 1960), p. 5.

ROBIDOUX, Réjean, «Livres en français (...) Théâtre», University of Toronto Quarterly, vol. XXXIX, $\mathrm{n}^{\circ} 4$ (July 1970), pp. 441-444 (voir p. 443).

R(OYER), J(ean), «Le Théâtre populaire choyé par Québec ou vive versa», l'Action, vol. LXII, n' 19028 (14 mars 1970), p. 14.

RUDEL-TESSIER (Joseph), «Chez lui, à la campagne, il écrit... Pour cette pièce, Guy Dufresne a renoncé aux hommes de la mer», le Petit Journal, vol. XXXIII, n 39 (19 juillet 1959), p. 92.

RUDZIK, O. H. T., «Letters in Canada 1972. Fiction», University of Toronto Quarterly, vol. XLII, nº 4 (Summer 1973), pp. 343-357 (v. p. 354).

SABBATH, Lawrence, «Lavish Production of Canadian Play», The Montréal Star, vol. XCII, nº 24 (January 29, 1960), p. 15. 
SAUCIER, Pierre, «Comment Guy. Dufresne est venu au théâtre», Actualité ma paroisse, mars 1960, pp. 28-29.

SPENCER, Nigel, «Plays from Québec», Matrix, vol. I, $\mathrm{n}^{\circ} 1$ (Spring 1975); pp. 28-29.

, «Acting out Ourselves», Books in Canada, vol. II, $\mathrm{n}^{\circ} 1$ (January-February 1973), p: 28.

\section{Décembre}

(Anonyme), «Le théâtre aux Beaux Dimanches», Ici Radio-Canada, vol. XII, no 37 (9-15 septembre 1978), p. 5.

BASILE, Jean, «Décembre. Un exercice d'austérité», le Devoir, vol. LXIX, n² 294 (19 décembre 1978), p. 8.

COUSINEAU, Louise, "Sortir la télé des solutions d'il y a quinze ans», la Presse, vol. XCIV, no 189 (16 décembre 1978), p. D-16.

CUSSON, Normand, «Décembre est l'une des plus ambitieuses dramatiques de notre télévision», T.V. Hebdo, vol. XIX, n ${ }^{\circ} 21$ (16-22 décembre 1978), pp. 9A-10A.

HOULE, René, «Une dramatique documentaire émouvante et forte», Ici Radio-Canada, vol. XII, $n^{\circ} 51$ (16-22 décembre 1978), pp. 8-9.

\section{Deux-zéro-sonnez-quatre}

(Anonyme), «Nouveaux récits sur la vie dans un milieu agricole», la Semaine à Radio-Canada, vol. I, n 42 (29 juillet - 4 août 1951), p. 2.

\section{Dieu ayde au premier baron chrétien}

(Anonyme), «Images du Canada. Deux textes de Guy Dufresne», la Semaine à Radio-Canada, vol. XI, nº 3 (15-21 octobre 1960), p. 2.

\section{Docile}

(Anonyme), «Les arts cette semaine. Théâtre. Comédie canadienne», la Presse, vol. LXXXIV, ${ }^{\circ} 105$ (4 mai 1968), p. 32.

, «Docile, vendredi soir», la Presse, vol. LXXXIV, n 109 (8 mai 1968), p. 74 .

, "Guy Dufresne, une pièce. de théâtre (enfin!) après trois téléromans", la Patrie, vol. LXXXIX, n 19 (12 mai 1968), p. 41.

"Comédie pudiquement érotique», Montréal-matin, vol. XXXVIII, $\mathrm{n}^{\circ} 272$ (25 mai 1968), p. 18.

, «Le vocabulaire savoureux de Guy Dufresne», le Soleil, vol.

LXXI, n 134 (4 juin 1968), p. 18. 
juin 1968), p. 23.

, «Docile à Montréal jusqu'au 9», le Droit, vol. LVI, $n^{\circ} 62(8$ , «Docile», le Livre canadien, vol. III, $\mathrm{n}^{\circ} 158$ (1972).

, «Dès le 10 mai à la Comédie canadienne. Docile de Guy Dufresne», le Devoir, vol. LIX, n 105 (4 mai 1968), p. 17.

COURTEMANCHE, Gilles, «Guy Dufresne, auteur dramatique», programme de Docile, Montréal, Comédie canadienne, 1968, (n.p.).

DASSYLVA, Martial, «Docile, la petite 'geuse' (sic)», la Presse, vol. LXXXIV, no 111 (11 mai 1968), p. 2.

D'AUTEUIL, Georges-Henri, «Le théâtre (...) Docile», Relations, $\mathrm{n}^{\circ} 328$ (juin 1968), pp. 192-193.

DORÉ, Fernand, «La liberté sous les pommiers», le Magazine Maclean, vol. VIII, $n^{\circ} 4$ (avril 1968), p. 78.

FÉRAL, Josette, «Docile», Dictionnaire des ouvres littéraires du Québec, sous la direction de Maurice Lemire avec la collaboration de Gilles Dorion, André Gaulin, Alonzo Le Blanc, Aurélien Boivin, Roger Chamberland, Kenneth Landry et Lucie Robert, tome IV, 1960-1969, Montréal, Fides, 1984 (à paraître).

GODIN, Jean-Cléo, «Docile de Guy Dufresne», Livres et Auteurs québécois, 1972, p. 125.

HELLER, Zelda, "Gélinas Scores Double in Dufresne Play», The Gazette, vol. CXC (May 13, 1968), p. 35.

IRWIN, Joan, "Dufresne's Docile. Fortune Telling, Rural Style», The Montreal Star, vol. C, $\mathrm{n}^{\circ} 112$ (May 11, 1968), p. 57.

KATTAN, Naïm, «Le théâtre et les dramaturges à Montréal», Canadian Litérature, $\mathrm{n}^{\circ} 40$ (Spring 1969), pp. 43-48; reproduit dans William H. New (editor), Dramatists in Canada. Selected Essays, Vancouver, University of British Columbia Press, 1972, pp. 146-147.

L(ÉVY), B(ernard), "Théâtre. 'R'chale-moi donc pas!' Docile», SeptJours, vol. II, $\mathrm{n}^{\circ} 36$ (19-25 mai 1968), pp. 45-46.

MAILHOT, Laurent, "Chroniques. Le théâtre. Des missionnaires aux sauvages ou du sacré au sacrant», Études françaises, vol. VIII, $\mathrm{n}^{\circ} 4$ (novembre 1972), pp. 409-427, (v. pp. 412-413).

«Letters in Canada (...) Théâtre», University of Toronto

Quarterly, vol. XLII, nº 4 (Summer 1973), pp. 363-366 (v. p. 363).

MAJOR, André, "Comédie canadienne. Docile, la farce de Dufresne», le Devoir, vol. LIX, ${ }^{\circ} 112$ (13 mai 1968), p. 9.

PIAZZA, François, «Votre critique au théâtre. Docile», Écho-Vedettes, vol. VI, $n^{\circ} 19$ (25 mai 1968), p. 27. 


\section{Les Forges de Saint-Maurice (Voir aussi Ce maudit Lardier)}

(Anonyme), «En cours de production : les Forges de Saint-Maurice de Guy Dufresne», Ici Radio-Canada, vol. XV, n 23 (3-9 juin 1972), p. 9.

«Rivalité entre les familles Chaput et Godard», Ici RadioCanada, vol. VI, $\mathrm{n}^{\circ} 39$ (23-29 septembre 1972), p. 5.

, «Le bailleur de fonds des Forges vient constater les dégâts», Ici Radio-Canada, vol. VI, $\mathrm{n}^{\circ} 43$ (21-27 octobre 1972), p. 4.

"Il y a bal aux Forges dans un décor somptueux», Ici RadioCanada, vol. VIII, $\mathrm{n}^{\circ} 4$ (19-25 janvier 1973), pp. 8-9.

«L'arrivée d'un nouveau fondeur fera rebondir l'action des Forges de Saint-Maurice», Ici Radio-Canada, vol. VII, n 33 (11-17 août 1973), pp. 8-9.

, «Radio-Canada fait une large place aux dramatiques avec cinq téléromans et de nombreux téléthéâtres aux 'Beaux Dimanches'», Ici Radio-

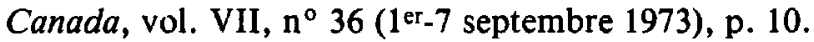

, «Le nouveau fondeur allumera-t-il le haut fourneau?», Ici Radio-Canada, vol. VII, $\mathrm{n}^{\circ} 39$ (22-28 septembre 1973), p. 3.

«Le haut fourneau des Forges de Saint-Maurice est enfin allumé et M. de Vézin danse», Ici Radio-Canada, vol. VII, $\mathrm{n}^{\circ} 50$ (8-14 décembre 1973), p. 15.

BIRON, Hervé, «Les Forges de Saint-Maurice de Mgr Albert Terrier. Pour se désintoxiquer des insignifiances de la télévision», le Jour, vol. II, $\mathrm{n}^{\circ} 79$ (4 juin 1975), p. 13.

CÔTÉ, Fernand, "Guy Dufresne nous dévoile la petite histoire des Forges de Saint-Maurice», Ici Radio-Canada, vol. VII, $\mathrm{n}^{\circ} 8$ (17-23 février 1973), $\mathrm{p}$. 7.

DAIGNEAULT, Claude, "Des Plouffe... aux Forges de Saint-Maurice», le Soleil, vol. LXXVI, $\mathrm{n}^{\circ} 214$ (9 septembre 1972), pp. 16-17.

DUFRESNE, Guy, «Des précisions de Guy Dufresne. Un peu d'histoire autour des Forges de Saint-Maurice (réponse à Hervé Biron)", le Jour, vol. II, $\mathrm{n}^{\circ} 98$ (28 juin 1975), p. 13.

LALONDE, Claire, «Une entrevue avec Richard Martin, le réalisateur des Forges...", Ici-Radio-Canada, vol. VII, nº 29 (14-20 juillet 1973), p. 7.

LEGAULT, Réjean, "Les Forges de Saint-Maurice: une histoire non forgée», T:V. Hebdo, vol. X, no 32 (18-24 avril 1970), pp. 16-17.

LESSONINI, Guy, «Les Forges de Saint-Maurice : ce n'est pas une histoire à l'eau de rose», T.V. Hebdo, vol. XI, n ${ }^{\circ} 51$ (24-30 janvier 1971), pp. 12-16. 
RIVARD, Yolande, "Branle-bas sur les lieux de tournage des Forges de Saint-Maurice», Ici Radio-Canada, vol. VI, n 32 (5-11 août 1972), p. 8

RUDEL-TESSIER (Joseph), «Guy Dufresne : l'auteur qui ne se fait pas la vie facile», la Presse (supp. télévision), vol. LXXXVIII, $\mathrm{n}^{\circ} 135$ (15-22 juillet 1972), p. 3, p. 9 et p. 13.

, «Cette histoire des Forges de Saint-Maurice ne s'arrêtera que dans... 143 ans», la Presse (supp. télévision), vol. LXXXVIII, nº 219 (21-28 octobre 1972), pp. 25-29.

\section{L'T̃e-aux-pormmes}

(Anonyme), "L'Île-aux-pommes de Guy Dufresne», la Semaine à RadioCanada, vol. III, $\mathrm{n}^{\circ} 3$ (26 octobre - 1er novembre 1952), p. 8.

\section{Le Jeu sur la presqu'île}

(Anonyme), «Jeu scénique au Parc Lafontaine, le 22 juin», la Patrie, vol. LXXIV, $\mathrm{n}^{\circ} 98$ (21 juin 1952), p. 56.

, «Jeu sur la presqu'île. Succès de la fête du centre paroissial de l'Immaculée-Conception», la Presse, vol. LXVIII, $\mathrm{n}^{\circ} 211$ (23 juin 1952), p. 9.

\section{Johanne et ses vieux}

(Anonyme), "Guy Dufresne met en scène des septuagénaires», Ici RadioCanada, vol. X, n ${ }^{\circ} 44$ (23-29 octobre 1976), p. 5.

BASILE, Jean, «Télévision. Johanne et ses vieux: une réussite», le Devoir, vol. LXVIII, $\mathrm{n}^{\circ} 250$ (29 octobre 1976), p. 12.

BURELLE, Guy, «Six nouvelles émissions mises à la disposition du public», Ici Radio-Canada, vol. XII, n' 29 (15-21 juillet 1978), p. 8.

COUSINEAU, Louise, «Johanne et ses vieux : oui la télévision peut être superbe», la Presse, vol. XCII, $\mathrm{n}^{\circ} 256$ (25 octobre 1976), p. D-13.

DESLANDES, Pierrette, "Johanne et ses vieux, le drame de la vieillesse», T.V. Hebdo, vol. XVIII, ${ }^{\circ} 13$ (23-29 octobre 1976), pp. 10A-11A.

\section{Kah-na-wio (Voir aussi Wahta)}

( Anonyme), «Kanawio (sic) de Guy Dufresne», la Semaine à RadioCanada, vol. XII, n 3 (14-20 octobre 1961), p. 3.

1962), p. 65 . "Kanawio», T.V. Hebdo, vol. II, $\mathrm{n}^{\circ} 37$ (28 avril - 4 mai «L'histoire de trois familles iroquoises. Kanawio», T.V. Hebdo, vol. II, $\mathrm{n}^{\circ} 12$ (4-10 novembre 1962), pp. 55-57.

BENOÎT, Fernand, «Les téléromans peuvent-ils traiter des problèmes adultes?», T.V. Hebdo, vol. II, nº 21 (6-12 janvier 1962), pp. 55-58. 
CÔTÉ, Fernand, «Jose Rodriguez 'l'insondable'», T.V. Hebdo, vol. I, $\mathrm{n}^{\circ}$ 39 (12-18 mai 1962), pp. 59-60.

LEROUX, Ulric, «La télévision. Kanawio, l'histoire de trois familles iroquoises», le Droit, vol. XLIX, nº 242 (18 octobre 1961), p. 11.

TAINTURIER, Jean, «La télévision. Music-hall, Bobino, téléromans», le Devoir, vol. LII, $\mathrm{n}^{\circ} 270$ (18 novembre 1961), p. 15.

\section{Kébec}

(Anonyme), «Kébec», la Semaine à Radio-Canada, vol. IX, $\mathrm{n}^{\circ} 5$ (1 $^{\mathrm{er}-7}$ novembre 1958), p. 11.

COUCKE, Paul, «Traits de plume. Kébec de Guy Dufresne», la Patrie, vol. XXIV, n ${ }^{\circ} 45$ (9 novembre 1958), p. 127.

VALOIS, Marcel (pseudonyme de Jean DUFRESNE), «Sons et images. Pages d'histoire à Kébec"), la Presse, vol. LXXV, $\mathrm{n}^{\circ} 17$ (4 novembre 1958), p. 36 .

\section{Marie Hurdouil s'est noyée}

(Anonyme), «Marie Hurdouil s'est noyée», la Semaine à Radio-Canada, vol. $\mathrm{V}, \mathrm{n}^{\circ} 45$ (14-20 août 1955), pp. 8, 7 .

\section{Mesure de guerre}

CHALVIN, Michel, «Après Mesure de guerre, un téléroman? 'Je me sens prêt à repartir' (Guy Dufresne)», la Semaine à Radio-Canada, vol. X, ${ }^{\circ} 31$ (30 avril - 6 mai 1960), p. 4.

COUCKE, Paul, «L'auteur de Cap-aux-sorciers (sic) reviendrait dans un téléroman», la Patrie du dimanche, vol. XXVI, $\mathrm{n}^{\circ} 18$ (1 ${ }^{\mathrm{er}}$ mai 1960), p. 118.

HÉNAULT, Gilles, «Fresque historique de Guy Dufresne», le Devoir, vol. LI, n 97 (3 mai 1960), p. 7.

LEROUX, Ulric, «La télévision. Le vrai caractère de Dollard dépeint à Mesure de guerre», le Droit, vol. XLVIII, nº 104 (4 mai 1960), p. 12.

\section{Mon village sur la mer (Voir aussi Cap-aux-Sorciers)}

(Anonyme), «Mon village sur la mer. Guy Dufresne fait revivre, à la radio, les personnages de Cap-aux-Sorciers», la Semaine à Radio-Canada, vol. XI, $n^{\circ} 22$ (25 février - 3 mars 1961), p. 6.

\section{Nicolas Dumetz}

(Anonyme), "La semaine à la télévision. Une pièce de Guy Dufresne à l'affiche du théâtre d'été», la Semaine à Radio-Canada, vol. IV, no 47 (29 août - 4 septembre 1954), p. 8.

ROBERT, Lucette, «Nicolas Dumetz», la Revue populaire, vo. XLVII, $\mathrm{n}^{\circ}$ 10 (octobre 1954), p. 9. 


\section{Sacrifice}

(Anonyme), «Le concours littéraire de Radio-Canada», Radiomonde, vol: VIII, $\mathrm{n}^{\circ} 10$ (16 février 1946), p. 2.

\section{Septième nord}

(Anonyme), "Septième nord est signé Guy Dufresne», T.V. Hebdo, vol. IV, $n^{\circ} 9$ (12-18 octobre 1963$)$, p. 59.

, «Le travail d'équipe, principal artisan de Septième nord», la Semaine à Radio-Canada, vol. XIV, nº 6 (2-8 novembre 1963), pp. 6-7 «Le Pain du jour et Septiène nord», la Semaine à RadioCanada, vol. XIV, $\mathrm{n}^{\circ} 50$ (5-11 septembre 1964), p. 8.

"Guy Dufresne conçoit Septième nord sous les pommiers", Divertissement, vol. I, $\mathrm{n}^{\circ} 6$ (septembre 1966), pp. 10-11.

(BEAUDRY)-BÉCHARD, Marguerite, «Trois nouvelles émissions dramatiques à la télévision", la Semaine à Radio-Canada, vol. XIII, ñ $^{\circ} 51$ (14-20 septembre 1963), pp. 4-5.

, «Monique Miller aime être dirigée», T.V. Hebdo, vol: V, $\mathrm{n}^{\circ}$ 18 (12-18 décembre 1964), pp. 7-10.

COUCKE, Paul, «L'auteur de Cap-aux-Sorciers reviendrait dans un téléroman», la Patrie du dimanche, vol. XXVI, $\mathrm{n}^{\circ} 118$ (1 ${ }^{\mathrm{er}}$ mail 1960), p. 118.

DUFRESNE, Georges, "Ces femmes vous ressemblent-elles?», Châtelaine, vol: VII, nº 2 (février 1966), p. 19.

JULIEN, Marie, «Télévision (...) Septième nord», Actualité, vol. VI, n ${ }^{\circ} 6$ (juin 1965), p. 38.

SIMARD, Pierre, «L'humour de l'auteur fait de Septième nord un téléroman de classe», Télé-Radiomonde, vol. XVII, $\mathrm{n}^{\circ} 43$ (17 septembre 1966), p. 9.

\section{Les Traitants}

(Anonyme), «Les livres en bref. Les Traitants», le Québec en bref, vol. III, $\mathrm{n}^{\circ} 11$ (novembre 1969), p. 22.

, «Les Traitants au Théâtre du dimanche», T.V. Hebdo, vol. I, $\mathrm{n}^{\circ} 22$ (12-18 janvier 1961), pp. 19-20.

"Théâtre du dimanche. Une enquête royale sous le Régime français. Les Traitants de Guy Dufresne», la Semaine à Radio-Canada, vol. XI, $n^{\circ} 16$ (14-20 janvier 1961), p. 16.

, «Les Traitants de Guy Dufresne à l'affiche du Nouveau Monde», le Soleil, vol. LXXII, nº 47 (22 février 1969), p. 33. 
DASSYLVA, Martial, "Théâtre. Au TNM, enquête royale sur les méfaits de l'eau-de-vie. Les Traitants», la Presse, vol. LXXXV, $n^{\circ} 51$ (1 $^{\text {er }}$ mars 1969), p. 24.

, «La turbulence d'une vraie cour de village», la Presse, vol. LXXXV, $\mathrm{n}^{\circ} 52$ (3 mars 1969), p. 25; reproduit dans Un théâtre en effervescence. Critiques et chroniques 1965-1972. Montréal, Éditions la Presse, 1975, 283 p. (v. pp. 81-83).

D'AUTEUIL, Georges-Henri, «Le théâtre (...) Les Traitants», Relations, $\mathrm{n}^{\circ} 337$ (avril 1969), p. 121.

FÉRAL, Josette, «Les Traitants», Dictionnaire des oeuvres littéraires du Québec, sous la direction de Maurice Lemire, avec la collaboration de Gilles Dorion, André Gaulin, Alonzo Le Blanc, Aurélien Boivin, Roger Chamberland, Kenneth Landry et Lucie Robert, tome IV, 1960-1969, Montréal, Fides, 1984 (à paraître).

GARON, Jean, «L'histoire en tant que théâtre conservateur et inoffensif», le Soleil, vol. LXXII, ${ }^{\circ} 61$ (11 mars 1969), p. 27.

GERMAIN, Jean-Claude, «Les Traitants : à la manière de Kriegoff, W.H. Drummond, Alan Mills, Jacques Labrecque et autres good-fellows...», Dimensions, vol. VI, $\mathrm{n}^{\circ} 5$ (mai 1969), pp. 7-10.

GODIN, Jean-Cléo, «Les Traitants», Livres et auteurs québécois, 1969, pp. 76-77.

H(ELLER), Z(elda), "Les Traitants. Social Problem in New France», The Montréal Star, vol. CI, n 52 (March 3, 1969), p. 12.

HÉNAULT, Gilles, «Le Théâtre du dimanche à la télévision. Une remarquable reconstitution historique», le Devoir, vol. LII, $\mathrm{n}^{\circ} 13$ (17 janvier 1961), p. 6.

HOFFMAN, Pierre, "Théâtre canadien d'expression française et réalisation télévisée». Thèse de maîtrise ès arts, Montréal, Université de Montréal, 1970, ii,108,6 f.

KATTAN, Naïm, "Le théâtre et les dramaturges à Montréal», Canadian Literature, $\mathrm{n}^{\circ} 40$ (Spring 1969), pp. 43-48; reproduit dans William H. NEW (editor), Dramatists in Canada. Selected Essays, Vancouver, University of British Columbia Press, 1972, pp. 146-147.

KILGOUR, Don, "Guy Dufresne's Les Traitants staged by Théâtre du Nouveau Monde», The Gazette, vol. CXCI (March 3, 1969), p. 10.

LEROUX, Ulric, «Le télévision. Les Traitants, tranche piquante de notre histoire», le Droit, vol. XLIX, nº 14 (18 janvier 1961), p. 12.

MAJOR, André, «Théâtre. Les Traitants de Guy Dufresne», le Devoir, vol. LX, n 52 (4 mars 1969), p. 10. 
P(IAZZA), F(rançois), «La page de la critique. Théâtre. Les Traitants», Échos-Vedettes, vol. VII, $\mathrm{n}^{\circ} 9$ (15 mars 1969), p. 26.

ROBIDOUX, Réjean, "Livres en français (...) Théâtre», University of Toronto Quarterly, vol. XXXIX, ${ }^{\circ} 4$ (July 1970), pp. $441-444$ (v. p. 443).

ROYER, Jean, «Les Traitants au TNM», l’Action, vol. LXII, n 18784 (15 mars 1969), p. 15.

\section{La Veilleuse}

(Anonyme), «Conte de Noël de Guy Dufresne», la Semaine à RadioCanada, vol. V, in ${ }^{\circ} 11$ (19-25 décembre 1954), p. 12.

\section{Vie de Lord Selkirk}

(Anonyme), «Guy Dufresne décrira une généreuse entreprise», la Semaine d̀ Radio-Canada, vol. II, $\mathrm{n}^{\circ} 21$ (2-8 mars 1952), p. 8.

\section{Wahta (Voir aussi Kah-na-wio)}

BEAUDRY-BÉCHARD, Marguerite, «Une œuvre inédite de Guy Dufresne», la Semaine à Radio-Canada, vol. XIV, $\mathrm{n}^{\circ} 26$ (21-27 mars 1964), pp. 14-15. 